\title{
Propiedades Psicométricas de la Versión en Español del Test de Compromiso con la Vida en Personas con Discapacidad Física
}

\author{
Psychometric Properties of the Spanish Version of the Life Engagement Test in \\ People with Physical Disability
}

\author{
Sandra Lima-Castro ${ }^{1}$, Paúl Arias-Medina ${ }^{2}$, Vanessa Blanco ${ }^{3}$ y Fernando L. Vázquez ${ }^{4}$
}

\begin{abstract}
Resumen
El objetivo de este estudio fue analizar las propiedades psicométricas y la estructura factorial de la versión en español del Test de Compromiso con la Vida (Life Engagement Test; LET) en personas con discapacidad física. El estudio involucró una muestra aleatoria de 479 adultos ecuatorianos con discapacidad física (56.78\% mujeres, edad media 52.29 años). Se dividió la muestra aleatoriamente en dos grupos, para el análisis factorial exploratorio (AFE) y el análisis factorial confirmatorio (AFC). La consistencia interna fue de .81. Se encontró un único factor que explicó el $55.41 \%$ de la varianza total. Se encontraron correlaciones significativas del propósito en la vida con los diferentes dominios de la calidad de vida relacionada con la salud y con resiliencia. La LET constituye un instrumento fiable y válido para evaluar el propósito en la vida en personas con discapacidad física.
\end{abstract}

Palabras clave: propósito en la vida, compromiso con la vida, LET, propiedades psicométricas, análisis factorial confirmatorio, discapacidad física

\begin{abstract}
The objective of this study was to analyze the psychometric properties and factor structure of the Spanish version of the Life Engagement Test (LET) in people with physical disabilities. The study involved a random sample of 479 Ecuadorian adults with physical disabilities (56.78\% women, mean age 52.29 years). The sample was randomly divided into two groups, for exploratory factor analysis (EFA) and confirmatory factor analysis (CFA). Internal consistency was .81. A single factor was found that explained $55.41 \%$ of the total variance. Significant correlations of life purpose were found with different domains of health-related quality of life and resilience. The LET is a reliable and valid instrument to evaluate the purpose in life in people with physical disabilities.
\end{abstract}

Keywords: life purpose, life engagement, LET, psychometric properties, confirmatory factor analysis, physical disability

\footnotetext{
Los autores del trabajo agradecen a la Universidad de Cuenca por el apoyo y financiamiento recibido para el desarrollo de este estudio que formó parte del Proyecto de Investigación denominado "Calidad de vida en personas con discapacidad y variables psicosociales asociadas" ganador del XV Concurso organizado por la Dirección de Investigación de la Universidad de Cuenca [DIUC].

${ }^{1}$ Magíster en Psicoterapia Integrativa y Magíster en Psicología de la Salud, Doctoranda en Neurociencias y Psicología Clínica. Docente investigadora de la Universidad de Cuenca. Av. 12 de Abril y Agustín Cueva - Ciudadela Universitaria, Ecuador. Tel.: +593985739491. Correo: sandra.lima@ucuenca.edu.ec

${ }^{2}$ Máster en Estudios del Desarrollo. Docente Investigador de la Universidad de Cuenca. Av. 12 de Abril y Agustín Cueva - Ciudadela Universitaria, Ecuador. Tel.: +593999287616. Correo: paul.arias@ucuenca.edu.ec

${ }^{3}$ Doctor en Psicología, Profesor Ayudante Doctor, Universidad de Santiago de Compostela. Departamento de Psicología Evolutiva y de la Educación. Facultad de Psicología, Universidad de Santiago de Compostela, Campus Vida, 15782. Santiago de Compostela, España. Tel.: +34881813744. Correo: vanessa.blanco@usc.es

${ }^{4}$ Doctor en Psicología, Profesor Catedrátio de Universidad, Universidad de Santiago de Compostela. Departamento de Psicología Clínica y Psicobiología. Facultad de Psicología, Universidad de Santiago de Compostela, Campus Vida, 15782. Santiago de Compostela, España. Tel.: +34881813705. Correo: fernandolino.vazquez@usc.es

Revista Iberoamericana de Diagnóstico y Evaluación - e Avaliação Psicológica. RIDEP · No60 · Vol.3 · 15-26 · 2021

ISSN: 1135-3848 print /2183-6051online
} 


\section{Introducción}

El propósito en la vida se define como el grado en el que una persona está comprometida en actividades que son valiosas para ella (Scheier et al., 2006); este constructo lleva implícito un sentido de intencionalidad que guía el comportamiento (Barnes et al., 2007; Boyle et al., 2009) y la inversión de tiempo y energía para alcanzar un objetivo deseado (Ryff, 2014).

El estudio científico del propósito en la vida es de gran interés en los ámbitos de la medicina y la psicología (Brandstätter et al., 2012), debido a su potencial para predecir y promover una mejor salud (Kim et al., 2020; Roepke et al., 2014). Así, un mayor propósito en la vida se ha asociado con mayor longevidad (Hill \& Turiano, 2014); mejor calidad de vida (Jim et al., 2006); un menor riesgo de desarrollar una enfermedad cardiovascular (Kim et al., 2019), una discapacidad física (Mota et al., 2016), o de sufrir deterioro cognitivo (Boyle et al., 2010); una menor mortalidad (Tomioka et al., 2016); y una mayor implicación en la realización de actividad física (Hooker \& Masters, 2016). Por el contrario, la ausencia de propósito en la vida resulta en un vacío existencial (Crumbaugh \& Maholick, 1981) que, si no es resuelto, puede tener efectos adversos importantes en el bienestar físico y psicológico (Wrosch et al., 2003), asociándose incluso a una mayor mortalidad (Boyle et al., 2009).

El efecto positivo del propósito en la vida sobre la salud puede deberse a su efecto de amortiguación sobre la respuesta fisiopatológica a los estresores psicosociales y la discapacidad (Cohen et al., 2016; Yeung \& Breheny, 2019). Tener un mayor propósito en la vida promueve la flexibilidad psicológica y la resiliencia para hacer frente a las demandas, obstáculos y desafios (Hill et al., 2018; McKnight \& Kashdan, 2009), por lo que puede constituir un recurso psicológico protector importante contra el estrés (Lee et al., 2020; Pulopulos \& Kozusznik, 2018).

En la trayectoria vital de una persona existen muchas situaciones potencialmente estresantes en las que debe abandonar actividades que solían gustarle y llenar su vida de sentido (p.ej., una jubilación no deseada), y en ese contexto, debe encontrar actividades alternativas significativas en las que implicarse (Scheier et al., 2006). Una de estas situaciones estresantes, que plantea retos importantes y demanda un importante esfuerzo de adaptación, es tener una discapacidad física (Molton \& Yorkston, 2017). Las personas que la sufren frecuentemente experimentan dolor crónico (Carter et al., 2012), limitaciones en la participación y el apoyo social (Craig et al., 2015) y otras dificultades para mantener la continuidad de actividades y objetivos que llenan la vida de propósito, lo que requeriría buscar alternativas para satisfacer esta necesidad (Scheier \& Carver, 2001). Las definiciones de discapacidad han señalado el importantísimo rol de la interrupción de actividades significativas como uno de los mecanismos que relacionan la discapacidad con resultados negativos en la salud mental (World Health Organization [WHO], 2011). De hecho, la investigación ha encontrado que esa interrupción de la participación en actividades valoradas que puede ocurrir en el curso de una discapacidad puede precipitar la desrregulación de los estados afectivos (Meeks \& Depp, 2003; Ormel et al., 2002), y que la búsqueda de significado es una forma de ajustarse y enfrentarse a la realidad de la discapacidad para evitar consecuencias psicológicas negativas (Thompson et al., 2003).

Considerando la relevancia de la evaluación del constructo propósito en la vida, actualmente existen diversos instrumentos disponibles (Brandstätter et al., 2012). Sin embargo, las medidas más utilizadas presentan limitaciones. En primer lugar, su escasa sensibilidad al cambio, lo que dificulta la evaluación de la evolución en el propósito en la vida a medida que avanza el curso de una enfermedad degenerativa o mortal (Scheier et al., 2006). En segundo lugar, algunos instrumentos incluyen ítems que evalúan tanto el propósito en la vida como otros constructos conceptualmente relacionados pero distintos; por ejemplo, la Prueba de Propósito en la Vida (Purpose in Life Test [PIL]; Crumbaugh y Maholick (1964), ha sido cuestionada porque contiene ítems que evalúan el afecto positivo y negativo (Zika \& Chamberlain, 1992), estado de ánimo positivo (King \& Hicks, 2021), la vitalidad, y el sentido de responsabilidad (Steger et al., 2006); o el Índice de Perspectiva Vital (Life Regard Index [LRI]; Battista \& Almond, 1973), por incluir cuestiones que evalúan la realización 
emocional (Steger \& Kashdan, 2013; Steger et al., 2006). En tercer lugar, la estructura factorial de estas escalas presenta escasa consistencia (Brandstätter et al., 2012). Por último, instrumentos ampliamente utilizados como el PIL y la subescala de Propósito en la Vida de la Escala de Bienestar Psicológico de Ryff (Ryff \& Keyes, 1995) contienen ítems en retrospectiva que pueden confundir la evaluación del propósito actual con la percepción histórica de en qué medida han sido alcanzados los objetivos vitales (Karniol \& Ross, 1996; Pavot et al., 1998).

Para solventar dichas limitaciones se desarrolló el Test de Compromiso con la Vida (Life Engagement [LET]; Scheier et al., 2006). Este instrumento ha sido ampliamente utilizado en numerosos estudios científicos en el área de la salud. Entre sus ventajas destacan la brevedad en su aplicación y la ausencia de solapamiento de sus ítems con otras medidas relacionadas. Su versión original en inglés fue desarrollada con ocho muestras de EEUU, consistentes en adultos jóvenes de la comunidad ( $n=193)$, pacientes mujeres con osteoartritis $(n=183)$, sus esposos varones $(n=168)$, mujeres de mediana edad de la comunidad $(n=378)$, mujeres con un cáncer de pecho en un estadío temprano $(n=198)$, mujeres con un cáncer de pecho en estadío tardío $(n=86)$, y dos muestras de estudiantes universitarios $(n=359$ y $n=511$, respectivamente). Se encontró una estructura unifactorial, buena consistencia interna, y estabilidad temporal moderada durante aproximadamente cuatro meses ( $r=.61$ a .76$)$ (Scheier et al., 2006). Asimismo, presentó adecuada validez convergente, mostrando correlaciones significativas con autoestima, afecto hostil, los cinco grandes rasgos de personalidad (apertura a la experiencia, responsabilidad extraversión, amabilidad y neuroticismo), salud mental, apoyo social y satisfacción con la vida; y una mejor validez discriminante que la subescala de Propósito en la de Vida de la Escala de Bienestar Psicológico (Scheier et al., 2006).

Además, del trabajo original se han realizado otros estudios de validación, encontrándose entre los más relevantes los realizados por Pearson et al. (2013) en Australia y Sumi (2018) en Japón. En relación con el primero, sus propiedades psicométricas se analizaron en una muestra aleatoria de 545 adultos australianos, con edades de entre 55 y 94 años (Pearson et al., 2013), encontrando que la escala tenía una consistencia interna sólida sobre un intervalo de medición de 12 meses ( $\alpha \mathrm{s}=.84$ y .82 en $\mathrm{T} 1 \quad \mathrm{y} \quad \mathrm{T} 2$, respectivamente), y confirmando la estructura de un factor. En cuanto al segundo, se analizaron en dos muestras de Japón 409 estudiantes universitarios y 439 adultos trabajadores (Sumi, 2018); el análisis factorial exploratorio y confirmatorio apoyaron la estructura esperada de un factor; asimismo, se halló una consistencia interna aceptable ( $\alpha \mathrm{s}=.82$ a .86$)$, y se encontraron correlaciones con las puntuaciones de medidas de bienestar hedónico y eudaimónico.

A pesar de la importancia del propósito en la vida como determinante de la salud, no existen, en nuestro conocimiento, trabajos previos que hayan examinado las propiedades psicométricas del Test de Compromiso con la Vida en personas con discapacidad física; asimismo, ningún trabajo previo ha desarrollado y analizado las propiedades psicométricas de una versión en español de esta escala.

Por lo tanto, el objetivo de esta investigación fue analizar las propiedades psicométricas y la estructura factorial de la versión en español del Test de Compromiso con la Vida en una muestra de adultos ecuatorianos con discapacidad física.

\section{Método}

\section{Participantes}

Se empleó un diseño transversal. La muestra fue seleccionada por muestreo aleatorio simple sobre el listado de 9.740 personas con discapacidad física inscritas en el Registro Nacional de Discapacidades del Consejo Nacional para la Igualdad de Discapacidades (CONADIS) del Cantón Cuenca (Ecuador).

Los criterios para participar en el estudio incluyeron: (a) residir en el cantón Cuenca, (b) tener un rango de edad de entre 35 y 65 años, (c) presentar una discapacidad física congénita o adquirida (d) estar inscrito en el Registro Nacional de Discapacidades, y (e) comprometerse a participar en la evaluación. Se excluyó del estudio a aquellas personas que: (a) no dieron su consentimiento informado; o (b) presentaban cualquier dificultad en la comunicación (ej. no saber leer ni escribir) o condición que hiciese 
imposible la evaluación (ej., discapacidad intelectual, deterioro cognitivo significativo, deficiencia visual severa).

La tasa de respuesta fue del $82.0 \%$. Se invitó a participar al estudio a 667 personas con discapacidad física, de las que 120 se negaron a participar y 68 fueron excluidas por no cumplir los criterios de inclusión y exclusión. La muestra final quedó constituida por 479 participantes (56.78\% varones), de edades comprendidas entre los 35 y los 65 años $(M=52.29, D T=8.44)$ (Ver Tabla 1).

Todos los participantes fueron informados de la naturaleza del estudio y dieron su consentimiento informado. La participación fue voluntaria, sin incentivos económicos o de otro tipo. El estudio se realizó de acuerdo con la Declaración de Helsinki, y fue aprobado por el Comité de Bioética de la Universidad de la Universidad San Francisco de Quito (código 2017-104E).

\section{Instrumentos}

Cuestionario de las características de los participantes: Se elaboró un cuestionario ad hoc para obtener información sobre las siguientes variables: sexo, edad, pareja, etnia, ingresos mensuales y situación laboral.

Test de Compromiso con la Vida (Life Engagement Test [LET]; Scheier et al., 2006). Se trata de un test constituido por 6 ítems diseñados para medir el propósito en la vida. Cada ítem es evaluado en una escala Likert de 5 puntos que va desde 1 (totalmente en desacuerdo) a 5 (totalmente de acuerdo). Tres ítems se encuentran en dirección positiva (ítems 2,4 y 6 ) y otros tres en dirección negativa (ítems 1,3 y 5). El rango oscila de 6 a 30, indicando mayores puntuaciones un mayor compromiso con la vida. La consistencia interna de la versión original en inglés (alfa de Cronbach) osciló entre .72 y .87.

Además, se utilizaron además las siguientes escalas para valorar la validez convergente:

Escala de Calidad de Vida: Para evaluar la calidad de vida se empleó la versión española del Cuestionario de Calidad de Vida (WHOQOLBREF; World Health Organization [WHO], Division of Mental Health, 1996). La escala está formada por 26 preguntas, incluyendo un ítem sobre la calidad de vida global y otro sobre la satisfacción con el estado de salud. Las 24 preguntas restantes se corresponden con los cuatro dominios específicos de la calidad de vida relacionada con la salud: Físico, Psicológico, Social y Ambiente. Cada ítem de la del WHOQOL-BREF se puntúa en una escala tipo Likert del uno a cinco y contiene 4 escalas de respuesta relacionadas con la intensidad ("Nada" [1] - "Extremadamente" [5]); capacidad ("Nada" [1] - "Totalmente o Extremadamente" [5]); frecuencia ("Nunca" [1] - "Siempre" [5]); y evaluación de estados o comportamientos ("Muy insatisfecho" [1] - "Muy satisfecho" [5])

(WHO, Division of Mental Health, 1996). Las puntuaciones de los dominios pueden ser convertidas según una tabla de corrección para hacerlas comparables. Los rangos de cada dominio oscilan de 0 a 100 (WHO, Division of Mental Health, 1996) y presentan un alfa de Cronbach entre .69 y .90 (Urzúa \& Jarne, 2008). En esta muestra la escala reporta un alfa de Cronbach .78 para el dominio físico, .79 para el dominio psicológico, .71 para el dominio relaciones sociales y $\quad .83$ para el dominio ambiente.

Escala Breve de Resiliencia (Brief Resilience Scale [BRS] Smith et al., 2008, versión española de Rodríguez-Rey et al., 2016): Es una escala de seis ítems tipo Likert en un rango desde el 1 (totalmente en desacuerdo) hasta el 5 (totalmente de acuerdo). La puntuación total oscila de 6 a 30 . Tres ítems se encuentran en dirección positiva (ítems 1,3 y 5) y otros tres en dirección negativa (ítems 2, 4 y 6). La versión española de este instrumento presenta una consistencia interna superior a .70 (Rodríguez-Rey et al., 2016). En esta muestra la escala reporta un alfa de Cronbach .86 .

\section{Procedimiento}

Se desarrolló una versión en español de la LET para ser empleada en personas con discapacidad física, siguiendo las normas generales de traducción y adaptación de tests (Guillemin et al., 1993; Hambleton \& Zenisky, 2011) y las recomendaciones de la International Test Commission (2017), incluyendo la retrotraducción (Brislin et al., 1973). Los ítems del test fueron traducidos del inglés al español de manera independiente por dos investigadores bilingües cuya lengua materna era el español, y 
posteriormente retro-traducidos por un traductor independiente, cuya lengua materna era el inglés y que no había estado expuesto a la versión original del instrumento en inglés. Un panel de expertos revisó la retro-traducción para analizar y corregir los fallos en la versión en español, retrotraduciéndola y reevaluándola tantas veces como fue necesario para obtener una versión española equivalente a la original en inglés. Se administró esta versión del instrumento a 30 personas adultas con discapacidad física residentes en Cuenca (Ecuador); para asegurar la comprensión del cuestionario se solicitó a cada participante informar acerca de dificultades que encontraban en la comprensión de cada una de las preguntas y posibles sugerencias. No fueron necesarias modificaciones adicionales. La muestra empleada en este procedimiento no fue incluida en el análisis de datos.

Los participantes fueron reclutados desde el mes de septiembre del 2017 a abril del 2018 por vía telefónica; en estas llamadas se les brindó una breve descripción del estudio para motivar su participación, se efectuó una valoración del cumplimiento de criterios de inclusión y exclusión, se informó de que la participación era voluntaria, garantizando el anonimato de los participantes, y se obtuvo el consentimiento para proceder a la aplicación de los instrumentos del estudio. Tres psicólogos entrenados con experiencia práctica de al menos 200 horas en el área de evaluación psicológica recogieron de forma heteroadministrada los datos sociodemográficos y las respuestas a la LET, la WHOQOL-BREF y la BRS. El entrenamiento de los evaluadores fue realizado por el investigador principal, experto en evaluación psicológica, y consistió en un entrenamiento específico teóricopráctico de 24 horas que incluía clases instructivas y juego de roles. La duración de la evaluación fue de aproximadamente 30 minutos.

\section{Análisis de Datos}

En primer lugar, se calcularon las frecuencias, porcentajes, medias y desviaciones típicas de las variables sociodemográficas, las puntuaciones de la LET y sus ítems, de la calidad de vida y la resiliencia, tanto para la muestra total como para las dos submuestras. Para analizar las diferencias en compromiso vital en función de las características sociodemográficas se utilizó la prueba de suma de rangos de Wilcoxon, para la muestra total y las dos submuestras.

Para el análisis de fiabilidad se emplearon correlaciones de Pearson de sus ítems entre sí y de las puntuaciones de cada ítem con la puntuación total corregida, sobre la muestra total. La fiabilidad del instrumento se evaluó a través del alfa de Cronbach $(\alpha)$, y de la omega de McDonald

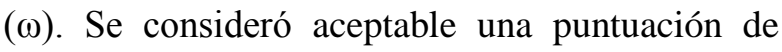
entre .7 y .8 , mientras que si era superior a .8 evidenciaba una alta consistencia interna (Cicchetti, 1994; Tavakol \& Dennick, 2011)

Para analizar la estructura factorial de la LET se dividió la muestra en dos grupos seleccionando aleatoriamente alrededor del $50 \%$ de los casos. Con la primera submuestra $(n=239)$, se llevó a cabo un análisis factorial exploratorio (AFE) para analizar las dimensiones subyacentes de la escala, con extracción de factores por mínimos cuadrados no ponderados y rotación varimax. Para determinar el número de factores a extraer, se empleó el número de ítems con un autovalor mayor de 1. La medida de adecuación de la muestra se realizó mediante el índice de KaiserMeyer-Olkin (KMO) y la matriz de correlaciones identidad se contrastó empleando el test de esfericidad de Bartlett.

Posteriormente, con la segunda submuestra $(n=240), \quad$ se realizó un análisis factorial confirmatorio para verificar la estructura factorial. La estimación utilizó una matriz de correlaciones policóricas. Para evaluar la bondad del ajuste del modelo se consideraron los siguientes índices y valores: el chi-cuadrado obtenido mediante una estimación de mínimos cuadrados ponderados diagonalizados (Diagonally Weighted Least Squares [DWLS]); el chi-cuadrado relativo, referido al cociente entre el valor del chi-cuadrado y los grados de libertad; el error cuadrático medio de aproximación (Root Mean Square Error of Approximation [RMSEA]); el índice GFI (Goodness of Fit Index); el índice ajustado de bondad de ajuste (Adjusted Goodness of Fit Index [AGFI]); el índice de ajuste comparativo (Comparative Fit Index [CFI]); el índice de ajuste normalizado (Normalized Fit Index [NFI]) y la raíz media cuadrática residual (Standardized Root Mean Square Residual [SRMR]). La ausencia de significación en las pruebas Chi-Cuadrado y 
Tabla 1. Características sociodemográficas y clínicas de la muestra

\begin{tabular}{|c|c|c|c|c|c|c|}
\hline \multirow[t]{2}{*}{ Variables } & \multicolumn{2}{|c|}{$\begin{array}{l}\text { muestra total } \\
\quad(n=479)\end{array}$} & \multicolumn{2}{|c|}{$\begin{array}{c}\text { submuestra AFE } \\
(n=239)\end{array}$} & \multicolumn{2}{|c|}{$\begin{array}{c}\text { submuestra AFC } \\
(n=240)\end{array}$} \\
\hline & $n$ & $\%$ & $n$ & $\%$ & $n$ & $\%$ \\
\hline \multicolumn{7}{|l|}{ Sexo } \\
\hline Mujer & 272 & 56.78 & 140 & 58.58 & 132 & 55.00 \\
\hline Hombre & 207 & 43.22 & 99 & 41.42 & 108 & 45.00 \\
\hline \multicolumn{7}{|l|}{ Edad } \\
\hline$M(D T)$ & \multicolumn{2}{|c|}{$52.29(8.44)$} & \multicolumn{2}{|c|}{$52.09(8.39)$} & \multicolumn{2}{|c|}{$52.49(8.51)$} \\
\hline Rango & \multicolumn{2}{|c|}{$35-65$} & \multicolumn{2}{|c|}{$35-65$} & \multicolumn{2}{|c|}{$36-65$} \\
\hline \multicolumn{7}{|l|}{ Pareja } \\
\hline No & 199 & 41.54 & 107 & 44.77 & 92 & 38.33 \\
\hline Sí & 280 & 58.46 & 132 & 55.23 & 148 & 61.67 \\
\hline \multicolumn{7}{|l|}{ Etnia } \\
\hline Mestizo & 454 & 94.78 & 228 & 95.40 & 226 & 94.17 \\
\hline Otros & 25 & 5.22 & 11 & 4.60 & 14 & 5.83 \\
\hline \multicolumn{7}{|l|}{ Ingresos mensuales } \\
\hline Menores o iguales a 375 & 262 & 54.70 & 139 & 58.16 & 123 & 51.25 \\
\hline Mayores a 375 & 217 & 45.30 & 100 & 41.84 & 117 & 48.75 \\
\hline \multicolumn{7}{|l|}{ Situación laboral } \\
\hline No trabaja & 297 & 62.00 & 142 & 59.41 & 155 & 64.58 \\
\hline Trabaja & 182 & 38.00 & 97 & 40.59 & 85 & 35.42 \\
\hline \multicolumn{7}{|l|}{ Dominio Físico } \\
\hline$M(D T)$ & \multicolumn{2}{|c|}{$47.67(18.86)$} & \multicolumn{2}{|c|}{$48.83(19.41)$} & \multicolumn{2}{|c|}{$46.52(18.26)$} \\
\hline Rango & \multicolumn{2}{|c|}{ 0-92.2 } & \multicolumn{2}{|c|}{$0-92.9$} & \multicolumn{2}{|c|}{ 3.6-92.9 } \\
\hline \multicolumn{7}{|l|}{ Dominio Psicológico } \\
\hline$M(D T)$ & \multicolumn{2}{|c|}{$59.02(19.85)$} & \multicolumn{2}{|c|}{$58.90(20.18)$} & \multicolumn{2}{|c|}{$59.14(19.58)$} \\
\hline Rango & \multicolumn{2}{|c|}{ 8.3-100 } & & & & \\
\hline Dominio Relaciones Socia & & & & & & \\
\hline$M(D T)$ & 56.15 & & 55.5 & & & $63)$ \\
\hline Rango & & & & & & \\
\hline Dominio Ambiente & & & & & & \\
\hline$M(D T)$ & 55.45 & & 56.0 & & & 04) \\
\hline Rango & 12 & & & & & \\
\hline Resiliencia & & & & & & \\
\hline$M(D T)$ & 19.2 & & 19. & & & 72) \\
\hline Rango & & & & & & \\
\hline
\end{tabular}

valores de RMSEA <.06, GFI o AGFI >.90, CFI o NFI cercanos a .95 y SRMR $<.08$ se consideraron indicadores de un buen ajuste (Browne \& Cudeck, 1993; Hair et al., 2006; Hu \& Bentler, 1999).

En relación con la validez convergente, se calcularon correlaciones de Pearson entre la LET y los cuatro dominios (físico, psicológico, social y ambiente) de la escala WHOQOL-BREF, y con la resiliencia evaluada a través de la escala BRS, empleando la muestra completa.

Todos los análisis estadísticos se realizaron con el software R versión 3.6.0.

\section{Resultados}

\section{Perfil de la muestra}

El $56.78 \%$ de las personas con discapacidad física fueron hombres, con una edad media de 52.29 años $(D T=8.44)$, y un rango de entre 35 y 65 años. La mayoría $(58.46 \%)$ tenían pareja, eran mestizos (94.78\%), tenían ingresos mensuales menores o iguales 375 dólares $(54.70 \%)$ y no trabajaban $(62.00 \%)$ (ver Tabla 1).

En relación con las variables clínicas, las puntuaciones para los diferentes dominios de WHOQOL-BREF fueron de $47.67 \quad(D T=18.86)$ para el dominio físico, $59.02(D T=19.85)$ para el dominio psicológico, $56.15(D T=22.11)$ para el dominio relaciones sociales, y $55.45(D T=17.58)$ para el dominio ambiente. La puntuación media de resiliencia fue de 19.29 ( $D T=4.8)$

No hubo diferencias significativas entre las puntuaciones medias para las variables sociodemográficas y clínicas entre los dos subgrupos. No hubo diferencias significativas en las puntuaciones del test LET en función de ninguna de las variables sociodemográficas, ni para la muestra total ni para los dos subgrupos.

\section{Consistencia Interna}

La puntuación media del test LET fue de 24.09 ( $D T=4.05$, rango=6-30). El porcentaje de los participantes que puntuaron 6 fue de $0.2 \%$ 
Tabla 2. Medias y Desviaciones Típicas (DT) de los Ítems, Frecuencias de las Puntuaciones y Correlación Elemento-Total Corregida $\left(r^{\text {tot }}\right)$ para cada Ítem $(n=479)$

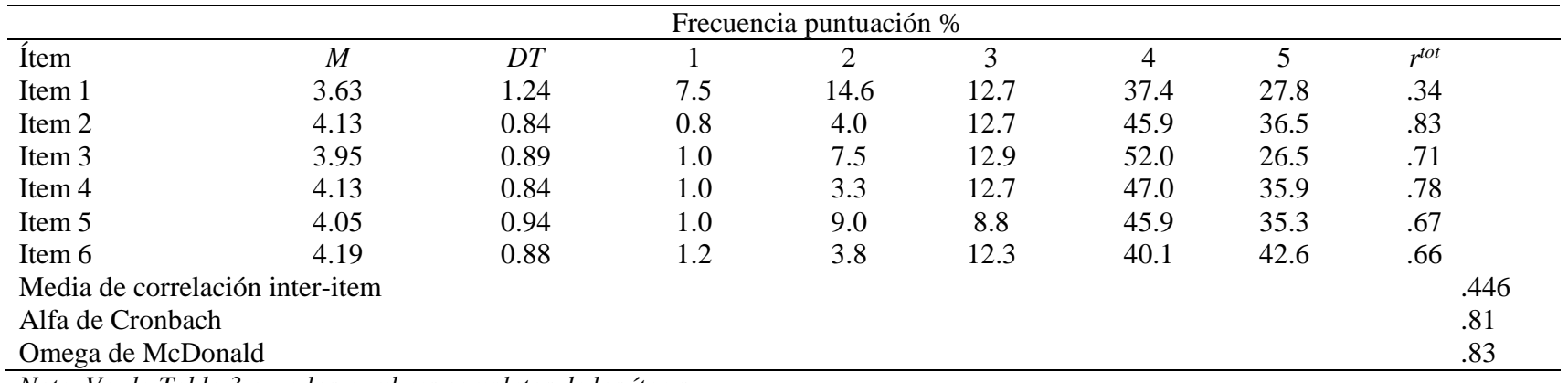

Nota. Ver la Tabla 3 para los nombres completos de los ítems.

Tabla 3. Cargas Factoriales de los Ítems del Test LET

\begin{tabular}{lc}
\hline Ítem & Factor 1 \\
\hline Mi vida no tiene suficiente propósito (sentido) (1) & .43 \\
Para mí, todas las cosas que hago valen la pena (2) & .88 \\
La mayor parte de lo que hago me parece trivial y sin importancia (3) & .77 \\
Valoro mucho mis actividades (4) & .84 \\
No me importan mucho las cosas que hago (5) & .75 \\
Yo tengo muchas razones para vivir (6) & .72 \\
\hline Nota. El número de los ítems se señala entre paréntesis &
\end{tabular}

Nota. El número de los ítems se señala entre paréntesis

(efecto suelo), mientras que el $9.4 \%$ obtuvo la puntuación máxima (efecto techo). Las medias de los ítems estuvieron en un rango de 3.63 para el ítem 1 (Mi vida no tiene suficiente propósito (sentido), y 4.19 para el ítem 6 (Yo tengo muchas razones para vivir). Los coeficientes de correlación elemento-total corregidos oscilaron de .34 para el ítem 1 (Mi vida no tiene suficiente propósito (sentido) a .83 para el ítem 2 (Para mí, todas las cosas que hago valen la pena), $\mathrm{y}$ todos fueron significativos $(p<.001)$. El coeficiente de correlación inter-ítem medio fue de .446, con un mínimo de .40 y un máximo de 55 . El alfa de Cronbach fue de $.81 \mathrm{y}$ el Omega de McDonald de .83.

\section{Análisis Factorial Exploratorio}

En el análisis factorial exploratorio, la medida de adecuación de la muestra de Kaiser-MeyerOlkin $(\mathrm{KMO}=.845)$ y el índice de esfericidad de Bartlett $\left(\chi^{2}(15)=561.854, p<.01\right)$ indicaron que la matriz de correlaciones podía ser factorizada. Se obtuvo un autovalor mayor que 1 que explicó el $55.03 \%$ de la varianza total. Este factor incluyó los 6 ítems, cuyas cargas factoriales oscilaron de .43 para el ítem 1 (Mi vida no tiene suficiente propósito [sentido]) y .88 para el ítem 2 (Para mí, todas las cosas que hago valen la pena) (Tabla 3).

\section{Análisis Factorial Confirmatorio}

A continuación, se llevó a cabo el AFC con el criterio de mínimos cuadrados ponderados diagonalizados. Los índices de ajuste para el modelo fueron $\chi^{2}(9)=36.39, \quad p<.001$, RMSEA $=.113$ GFI=.994, AGFI=.974, CFI=.993, $\mathrm{NFI}=.991, \quad \mathrm{SRMR}=.053$, indicando un ajuste suficiente, aunque moderado, a los datos. La Figura 1 muestra las cargas factoriales y errores de medida para cada ítem. Los pesos factoriales fueron satisfactorios, oscilando de .54 en el ítem 1 a .90 en el ítem 2 .

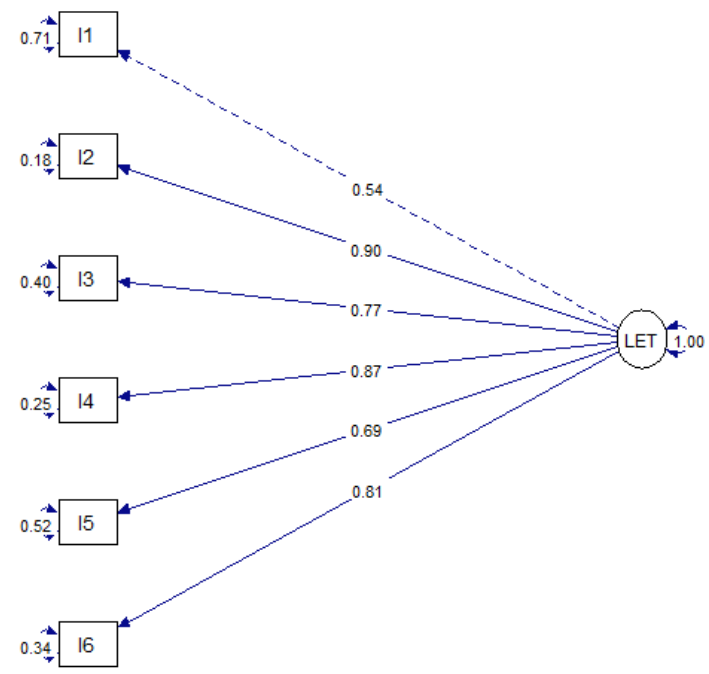

Figura 1. Resultados del Análisis Factorial Confirmatorio

\section{Validez convergente}

Como se muestra en la Tabla 4, con respecto a la validez convergente, se encontró una correlación directa significativa del propósito en la propósito en 
Tabla 4. Correlaciones entre la puntuación total del LET con Calidad de Vida y Resiliencia

\begin{tabular}{lccccc}
\hline Criterio & $\begin{array}{c}\text { dominio } \\
\text { físico }\end{array}$ & $\begin{array}{c}\text { dominio } \\
\text { psicológico }\end{array}$ & $\begin{array}{c}\text { dominio relaciones } \\
\text { sociales }\end{array}$ & $\begin{array}{c}\text { dominio } \\
\text { ambiente }\end{array}$ & Resiliencia \\
\hline Puntuación total LET & $.49^{* * *}$ & $.66^{* * *}$ & $.54 * * *$ & $.58^{* * *}$ & $.48 * * *$ \\
\hline Nota $: * * * p<.001$ & & & &
\end{tabular}

la vida con el dominio físico del WHOQOLBREF $(r=.49, p<.001)$, el dominio psicológico del WHOQOL-BREF $(r=.66, \quad p<.001)$, el dominio relaciones sociales del WHOQOLBREF ( $r=.54, p<.001)$, el dominio ambiente del WHOQOL-BREF $(r=.58, \quad p<.001), \quad$ y la resiliencia (BRS) $(r=.48 p<.001)$.

\section{Discusión y Conclusión}

Esta investigación tuvo como objetivo analizar las propiedades psicométricas y la estructura factorial del Test de Compromiso con la Vida en una muestra de adultos ecuatorianos con discapacidad física. La puntuación media para la escala LET fue de 24.09, dato similar al encontrado para la versión original por Scheier et al. (2006) en ocho muestras de EEUU, que fue de 25.1 para adultos jóvenes de la comunidad, 24.3 para pacientes mujeres con osteoartritis, 24.8 para sus esposos varones, 24.9 para mujeres de mediana edad de la comunidad, 25.3 para mujeres con un cáncer de pecho en un estadío temprano, 24.1 para mujeres con un cáncer de pecho en estadío tardío 24.1, y 23.6 y 24.4 para dos muestras de estudiantes universitarios, respectivamente. Además, estos hallazgos son consistentes con el estudio de Pearson et al. (2013) que informaron una media de 24.86 en T1 y $25.43 \mathrm{~T} 2$ en una muestra comunitaria de adultos mayores de 55 y 94 años sobre un intervalo de medición de 12 meses. Sin embargo, esta puntuación fue superior a la encontrada en el trabajo de Sumi (2018), que osciló entre 20.75 en estudiantes universitarios y 21.42 en adultos trabajadores japoneses. Este último hallazgo coincide con trabajos previos, que encontraron puntuaciones más bajas en propósito en la vida en muestras japonesas, con respecto a las americanas (Sato \& Tanaka, 1974; Steger et al., 2008); y diferencias en los objetivos deseables entre ambas culturas (Gore et al., 2009). La consitencia interna de la LET fue satisfactoria $(\alpha=.81, \omega=.83)$, con una media del coeficiente de correlación interítem de 446 , y coeficientes de correlación elemento-total corregidos $\geq .34$. La consistencia interna fue similar a la encontrada en el trabajo de Scheier et al. (2006) para pacientes mujeres con osteoartritis, y superior a la encontrada en la misma investigación para las muestras de adultos jóvenes de la comunidad, esposos de mujeres con osteoartritis, mujeres de mediana edad de la comunidad y estudiantes universitarios. Sin embargo, fue inferior a la encontrada en el estudio de Sumi (2018) para estudiantes universitarios y adultos trabajadores japoneses y en el trabajo de Pearson et al. (2013) para adultos mayores australianos.

El análisis factorial exploratorio identificó un único factor que explicó el 55.03\% de la varianza total. Este hallazgo es coherente con la estructura unifactorial de la versión original (Scheier et al., 2006), y sus versiones para población japonesa (Sumi, 2018) y adultos mayores australianos (Pearson et al., 2013). Además, el análisis confirmatorio mostró un ajuste suficiente, aunque moderado, a los datos.

En relación con la validez convergente, los resultados indicaron correlaciones con los diferentes dominios de calidad de vida evaluados mediante la escala WHOQOL-BREF. Este hallazgo concuerda con los resultados de un trabajo previo (Yeung \& Breheny, 2019), que encontró que el propósito en la vida puede ayudar a las personas mayores con discapacidad a lidiar con estresores de aparición temprana o cambios en la salud mental y física para mantener la calidad de vida en general. Asimismo, se evidenció una alta correlación entre el propósito en la vida y resiliencia, lo que concuerda con otras investigaciones (King \& Hicks, 2021).

Este estudio tiene importantes implicaciones para la investigación y la evaluación psicológica. Los hallazgos alcanzados permiten concluir que el test LET es un instrumento fiable y válido en población ecuatoriana con discapacidad, que presenta propiedades psicométricas adecuadas, 
similares a las alcanzadas en la versión original de la escala de Scheier et al. (2006). Contar con instrumentos validados para medir el propósito en la vida permite identificar a personas con alto $\mathrm{y}$ bajo propósito en la vida y diseñar evaluaciones e intervenciones novedosas desde una perspectiva integral de promoción para la salud mental (Castro, 2011; Lima-Castro et al., 2019). De hecho, el propósito en la vida, junto con otros componentes del bienestar, se ha convertido en el foco de múltiples estudios de intervención (de Jong et al., 2020; Chen et al., 2020).

Sin embargo, es necesario señalar también algunas limitaciones. En primer lugar, nuevas investigaciones deberían evaluar la adecuación de la estructura factorial obtenida en diferentes contextos culturales y muestras representativas de la población objeto de estudio. En segundo lugar, se requieren estudios adicionales que evalúen otras cualidades psicométricas importantes como la estabilidad temporal del instrumento (confiabilidad test-retest), validez discriminante y su robustez frente a sesgos de respuesta. Por otra parte, 120 personas se negaron a participar en el estudio pudiendo introducir sesgo en la respuesta. No obstante, esta tasa de respuesta, del $82 \%$, supera el $70 \%$ a partir del cual se considera que existe riesgo de producir estimaciones sesgadas (Singleton \& Straits, 2005).

En conjunto, los resultados de la presente investigación sugieren que el test LET tiene fiabilidad y validez adecuadas como medida de propósito en la vida en la población de personas con discapacidad física en Ecuador.

Agradecemos a M. F. Scheier el amable permiso para traducir el Test Compromiso con la Vida (Life Engagement [LET]].

\section{Referencias}

Barnes, L. L., Wilson, R. S., Bienias, J. L., de Leon, C. F. Mendes, K., H. N., Buchman, A. S., \& Bennett, D. A. (2007). Correlates of life space in a volunteer cohort of older adults. Experimental Aging Research, 33(1), 77-93. http://doi.org/10.1080/03610730601006420

Battista, J., \& Almond, R. (1973). The development of meaning in life. Psychiatry,
36.

http://doi.org/10.1080/00332747.1973.11023774

Boyle, P. A., Barnes, L. L., Buchman, A. S., \& Bennett, D. A. (2009). Purpose in life is associated with mortality among communitydwelling older persons. Psychosomatic Medicine, 71(5), 574-579. http://doi.org/10.1097/PSY.0b013e3181a5a7c0

Boyle, P. A., Buchman, A. S., Barnes, L. L., \& Bennett, D. A. (2010). Effect of a purpose in life on risk of incident Alzheimer disease and mild cognitive impairment in communitydwelling older persons. Archives of General Psychiatry, 67(3), 304-310.

http://doi.org/10.1001/archgenpsychiatry.2009 .208

Brandstätter, M., Baumann, U., Borasio, G. D., \& Fegg, M. J. (2012). Systematic review of meaning in life assessment instruments. Psycho-Oncology, 21(10), 1034-1052. http://doi.org/10.1002/pon.2113

Brislin, R. W., Lonner, W. J., \& Thorndike, E. M. (1973). Cross-cultural research methods. Wiley.

Browne, M. W., \& Cudeck, R. (1993). Alternative ways of assessing model fit. In I. K. Long (Ed.), Testing structural equation models (pp. 136-162). Sage.

Carter, G. T., Miró, J., Abresch, R. T., El-Abassi, R., \& Jensen, M. P. (2012). Disease burden in neuromuscular disease: the role of chronic pain. Physical Medicine and Rehabilitation Clinics of North America, 23(3), 719-729. http://doi.org/ 10.1016/j.pmr.2012.06.004

Castro, A. (2011). Las rutas de acceso al bienestar. Relaciones entre bienestar hedónico y eudaemónico. Un estudio en población argentina. Revista Iberoamericana de Diagnóstico y Evaluación - e Avaliação Psicológica, 37-57.

Chen, Y., Kim, E. S., Shields, A. E., \& VanderWeele, T. J. (2020). Antecedents of purpose in life: Evidence from a lagged exposure-wide analysis. Cogente Psychology, 7(1), 1825043. http://doi.org/10.1080/23311908.2020.1825043

Cicchetti, D. V. (1994). Guidelines, criteria, and rules of thumb for evaluating normed and standatized assesment in psychology. 
Psychological Assessment, 6(28), 284-290. http://doi.org/1.1037/1040-359.6.4.284

Cohen , R., Bavishi, C., \& Rozanski, A. (2016). Purpose in life and its relationship to all-cause mortality and cardiovascular events: A metaanalysis. Psychosomatic Medicine, 78(2), 122-133. http://doi.org/10.1097/PSY.0000000000000274

Craig, A., Nicholson, K., Guest, R., Tran, Y., \& Middleton, J. (2015). Adjustment following chronic spinal cord injury: Determining factors that contribute to social participation. British Journal of Health Psychology, 20(4), 807-823. http://doi.org/10.1111/bjhp.12143

Crumbaugh, J. C., \& Maholick, L. T. (1964). An experimental study in existentialism: The psychometric approach to Frankl's concept of noogenic neurosis. Journal of Clinical Psychology, 20, 200-207.

http://doi.org/10.1002/1097-

4679(196404)20:2<200::AID-

JCLP2270200203>3.0.CO;2-U

Crumbaugh, J. C., \& Maholick, L. T. (1981). Manual of instructions for the Purpose in Life Test. Institute of Logotherapy.

de Jong, E. M., Ziegler, N., \& Schippers, M. C. (2020). From shattered goals to meaning in life: Life crafting in times of the COVID-19 pandemic. Frontiers in Psychology, 11, 577708.

http://doi.org/10.3389/fpsyg.2020.577708

Gore, J. S., Cross, S. E., \& Kanagawa, C. (2009). Acting in our interests: Relational selfconstrual and goal motivation across cultures. Motivation and Emotion, 33(1), 75-87. http://doi.org/ 10.1007/s11031-008-9113-1

Guillemin, F., Bombardier, C., \& Beaton, D. (1993). Cross-cultural adaptation of healthrelated quality of life measures: Literature review and proposed guidelines. Journal of Clinical Epidemiology, 46(12), 1417-1432. http://doi.org/10.1016/0895-4356(93)90142-N

Hair, J., Black, W., Babin, B., Anderson, R., \& Tatham, R. (2006). Multivariate Data Analysis (6 ed.). Prentice Hall.

Hambleton, R. K., \& Zenisky, A. L. (2011). Translating and adapting tests for crosscultural assessments. In D. Matsumoto, \& F. van de Vijver (Eds.), Culture and psychology. Cross-cultural research methods in psychology (pp. 46-74). Cambridge University Press.

Hill, P. L., Sin, N. L., Turiano, N. A., Burrow, A. L., \& Almeida, D. M. (2018). Sense of purpose moderates the associations between daily stressors and daily well-being. Annals of Behavioral Medicine, 52(8), 724729. http://doi.org/10.1093/abm/kax039

Hill, P. L., \& Turiano, N. A. (2014). Purpose in life as a predictor of mortality across adulthood. Psychological Science, 25(7). http://doi.org/10.1177/0956797614531799

Hooker, S. A., \& Masters, K. S. (2016). Purpose in life is associated with physical activity measured by accelerometer. Journal of Health Psychology, 962-971. http://doi.org/10.1177/1359105314542822

Hu, L., \& Bentler, P. M. (1999). Cutoff criteria for fit indexes in covariance structure analysis: Conventional criteria versus new alternatives. Structural Equation Modeling: A Multidisciplinary Journal, 6(1), 1-55. http://doi.org/10.1080 / 10705519909540118

International Test Commission. (2017). The ITC Guidelines for Translating and Adapting [Segunda Edición]. Recuperado de International Test Commission: https://www.intestcom.org/files/guideline_test _adaptation_2ed.pdf

Jim, H., Purnell, J. Q., Richardson , S. A., GoldenKreutz, D., \& Andersen, B. L. (2006). Measuring meaning in life following cancer. Quality of Life Research, 15, 1355-1371. http://doi.org/10.1007/s11136-00-6-0028-6

Karniol, R., \& Ross, M. (1996). The motivational impact of temporal focus: Thinking about the future and the past. Annual Review of Psychology(47), 593-620.

Kim, E. S., Delaney, S. W., \& Kubzansky, L. D. (2019). Sense of purpose in life and cardiovascular disease: Underlying mechanisms and future directions. Current Cardiology Reports, 21(11), 135. http://doi.org/10.1007/s11886-019-1222-9

Kim, E. S., Shiba, K., Boehm, J. K., \& Kubzansky, L. D. (2020). Sense of purpose in life and five health behaviors in older adults. Preventive Medicine, 139, 106172. http://doi.org/10.1016/j.ypmed.2020.106172 
King, L. A., \& Hicks, J. A. (2021). The science of meaning in life. Annual Review of Psychology, 72(1), 561-584. http://doi.org/10.1146/annurev-psych-072420122921

Lee, J. E., Kahana, E., Kahana, B., \& Zarit, S. (2020). The role of goal and meaning in life for older adults facing interpersonal stress. Aging \& Mental Health, 1-11. http://doi.org/10.1080/13607863.2020.1849020

Lima-Castro, S., Peña-Contreras, E., Aguilar Sizer, M., Bueno Pacheco, A., \& Arias Medina, P. (2019). Aproximación a un Modelo de Bienestar en Adultos Ecuatorianos. Revista Iberoamericana de Diagnóstico y Evaluación - e Avaliação Psicológica. RIDEP, 2(51), 5-18. http://doi.org/10.21865/RIDEP51.2.01

McKnight, P. E., \& Kashdan, T. B. (2009). Purpose in life as a system that creates and sustains health and well-being: An integrative, testable theory. Review of General Psychology, 13(3), 242-251. http://doi.org/10.1037/a0017152

Meeks, S., \& Depp, C. A. (2003). Pleasant eventsbased behavioral intervention for depression in nursing home residents: A conceptual and empirical foundation. Clinical Gerontologist, 25(1-2), 125-148.

Molton, I. R., \& Yorkston, K. M. (2017). Growing older with a physical disability: A special application of the successful aging paradigm. The Journals of Gerontology, Series B, 72, 290299. http://doi.org/ 10.1093/geronb/gbw122

Mota, N. P., Tsai, J., Kirwin, P. D., Sareen, J., Southwick, S. M., \& Pietrzak, R. H. (2016). Purpose in life is associated with a reduced risk of incident physical disability in aging US military veterans. The American Journal of Geriatric Psychiatry, 24(9), 706-714. http://doi.org/10.1016/j.jagp.2016.03.004

Ormel, J., Rijsdijk, F. V., Sullivan, M., van Sonderen, E., \& Kempen, G. I. (2002). Temporal and reciprocal relationship between IADL/ADL disability and depressive symptoms in late life. The Journals of Gerontology Series B: Psychological Sciences and Social Sciences, 57(4), 338-347
Pavot, W., Diener, E., \& Suh, E. (1998). The temporal satisfaction with life scale. Journal of Personality Assessment, 70, 340-354.

Pearson, E. L., Windsor, T. D., Crisp, D. A., Butterworth, P., Pilkington, P. D., \& Anstey, K. J. (2013). Normative data and longitudinal invariance of the Life Engagement Test (LET) in a community sample of older adults. Quality of Life Research, 22(2), 327-331. http://doi.org/10.1007/s11136-012-0146-2

Pulopulos, M. M., \& Kozusznik, M. W. (2018). The moderating role of meaning in life in the relationship between perceived stress and diurnal cortisol. Stress, 21(3), 203-210. http://doi.org/10.1080/10253890.2018.1429397

Rodríguez-Rey, R., Alonso-Tapia, J., \& Hernansaiz-Garrido, H. (2016). Reliability and Validity of the Brief Resilience Scale (BRS) Spanish Version. Psychological Assessment, 28(5), 101-110. http://doi.org/10.1037/pas0000191

Roepke, A. M., Jayawickreme, E., \& Riffle, O. M. (2014). Meaning and health: A systematic review. Applied Research in Quality of Life, 9(4), 1055-1079. http://doi.org/10.1007/s11482-013-9288-9

Ryff, C. (2014). Psychological Well-Being Revisited: Advances in the Science and Practice of Eudaimonia. Psychotherapy and Psychosomatics, 83(1), 10-28. http://doi.org/10.1159/000353263

Ryff, C. D., \& Keyes, C. (1995). The structure of psychological well-being revisited. Journal of Personality and Social Psychology, 69, 719727.

Sato, F., \& Tanaka, H. (1974). An experimental study on the existential aspect of life: I. the cross-cultural approach to purpose in life. Tohoku Psychologica Folia, 33(1-4), 20-46.

Scheier, M. F., \& Carver, C. S. (2001). Adapting to cancer: The importance of hope and purpose. In Baum \& B. L. Andersen (Ed.), American Psychological Association. (pp. 1536). http://doi.org/10.1037/10402-002

Scheier, M. F., Wrosch, C., Baum, A., Cohen, S. M., Martire, L. M., Matthews, K. A., Sculz, R., \& Zdaniuk, B. (2006). The Life Engagement Test: Assessing purpose in life. Journal of Behavioral Medicine, 29(3), 291298. http://doi.org/10.1007/s10865-005-9044-1 
Singleton, R., \& Straits, B. C. (2005). Approaches to Social Research. Oxford University Press.

Smith, B. W., Dalen, J., Wiggins, K., Tooley, E., Christopher, P., \& Bernard, J. (2008). The brief resilience scale: Assessing the ability to bounce back. International Journal of Behavioral Medicine, 15, 194-200. http://doi.org/10.1080/10705500802222972

Steger, M. F., Frazier, P., Oishi, S., \& Kaler, M. (2006). The meaning in life questionnaire: Assessing the presence of and search for meaning in life. Journal of Counseling Psychology, 53(1), 80-93. http://doi.org/10.1037/0022-0167.53.1.80

Steger, M. F., \& Kashdan, T. B. (2013). The unbearable lightness of meaning: Well-being and unstable meaning in life. Journal of Positive Psychology, 103-115. http://doi.org/10.1080/17

Steger, M. F., Kawabata, Y., Shimai, S., \& Otake, K. (2008). The meaningful life in japan and the united states: Levels and correlates of meaning in life. Journal of Research in Personality, 42(3), 660-678. http://doi.org/ 10.1016/j.jrp.2007.09.003

Sumi, K. (2018). The Japanese translation of the Life Engagement Test: Reliability and construct validity in a college student population and a working adult population. Journal of Psychology \& Behavior Analysis, 4, 136. http://doi.org/10.15344/2018/2455-3867/136

Tavakol, M., \& Dennick, R. (2011). Making sense of Cronbach's alpha. International Journal of Medical Education, 53-55. http://doi.org/10.5116/ijme.4dfb.8dfd

Thompson, N. J., Coker, J., Krause, J. S., \& Henry, E. (2003). Purpose in life as a mediator of adjustment after spinal cord injury. Rehabilitation Psychology, 48(2), 100108. http://doi.org/10.1037/0090-5550.48.2.100

Tomioka, K., Kurumatani, N., \& Hosoi, H. (2016). Relationship of having hobbies and a purpose in life with mortality, activities of daily living, and instrumental activities of daily living among community. Journal of Epidemiology, 26(7), 361-370. http://doi.org/10.2188/jea.JE20150153.

Urzúa, A., \& Jarne, A. (2008). Calidad de vida y factores biopsicosociales en patologías médicas crónicas [Quality of life and biopsychosocial factors in chronic medical pathologies]. Terapía Psicológica, 26, 207214.

World Health Organization [WHO]. (2011). World report on disability 2011. Recuperado de https://apps.who.int/iris/handle/10665/44575

World Health Organization. Division of Mental Health. (1996). WHOQOL-BREF : Introduction, administration, scoring and generic version of the assessment: Field trial version. December 1996. Geneva. Recuperado de: Organization, World Health. http://www.who.int/iris/handle/10665/63529

Wrosch, C., Scheier, M. F., Miller, G. E., Schulz, R., \& Carver, C. S. (2003). Adaptive selfregulation of unattainable goals: Goal disengagement, goal re-engagement, and subjective well-being. Personality \& Social Psychology Bulletin, 29, 1494-1508.

Yeung, P., \& Breheny, M. (2019). Quality of life among older people with a disability: the role of purpose in life and capabilities. Disability and Rehabilitation, 1-11. http://doi.org/10.1080/09638288.2019.1620875

Zika, S., \& Chamberlain, K. (1992). On the relation between meaning in life and psychological well-being. British Journal of Psychology, 83, 133-145. http://doi.org/10.1111/j.20448295.1992.tb02429.x 